\title{
Columnar antiferromagnetic order of a MBene monolayer
}

\author{
Ilkay Ozdemir, ${ }^{1}$ Yelda Kadioglu, ${ }^{1}$ Yusuf Yüksel $\odot,{ }^{2}$ Ümit Akıncı, ${ }^{2}$ Olcay Üzengi Aktürk, ${ }^{3}$ \\ Ethem Aktürk ${ }^{1,4, *}$ and Salim Ciraci ${ }^{5, \dagger}$ \\ ${ }^{1}$ Department of Physics, Adnan Menderes University, 09100 Aydin, Turkey \\ ${ }^{2}$ Dokuz Eylul University, Faculty of Science, Physics Department, Tinaztepe Campus, 35390 Izmir, Turkey \\ ${ }^{3}$ Department of Electrical and Electronic Engineering, Adnan Menderes University, 09100 Aydin, Turkey \\ ${ }^{4}$ Physik Department E20, Technische Universität München, 85748 Garching, Germany \\ ${ }^{5}$ Department of Physics, Bilkent University, 06800 Ankara, Turkey
}

(Received 3 June 2020; revised 16 October 2020; accepted 5 April 2021; published 16 April 2021)

\begin{abstract}
First-principles density functional theory, combined with the Monte Carlo method, predicts that the $\mathrm{Fe}_{2} \mathrm{~B}_{2}$ monolayer of the MBene family has a stable columnar antiferromagnetic (AFM) ground state. Below the critical temperature, $T_{c}=115 \mathrm{~K}$ in equilibrium, the spins rotate by the same amount in every other column of $\mathrm{Fe}$ atoms, but they retain the same direction in the same column. Under applied tensile strains, $T_{c}$ and the order parameter can increase nonmonotonically. The onset of the columnar order can result in a transition from two dimension (2D) to $1 \mathrm{D}$ in magnetic, electronic, and conduction properties. The ordered magnetic state itself can be tuned by external magnetic field, whereby the columnar magnetic order changes to ferromagnetic order with a double hysteresis behavior. When terminated by Fluorine atoms, the columnar order changes to the AFM order with $T_{c}$ rising above room temperature. This situation is rather unusual and insofar is fundamental for a realistic, strictly 2D monolayer and can have critical consequences in spin conduction.
\end{abstract}

DOI: 10.1103/PhysRevB.103.144424

\section{INTRODUCTION}

Following the synthesis of graphene [1], several stable, strictly two-dimensional (2D) monolayer crystals with unprecedented electronic properties have been predicted and/or synthesized [2-19], but the progress to unveil their magnetic properties has been limited until recently. Despite previous theoretical studies using first-principles calculations $[12,20]$ which have predicted ferromagnetic (FM) order in transition metal dichalcogenide monolayers, the realization of magnetic ground state with $T_{c}>0 \mathrm{~K}$ was anticipated to be elusive due to spin fluctuations suppressing the ordered state in monolayers. However, recent observations on 2D van der Waals (vdW) structures [21,22] $\mathrm{CrI}_{3}$ and $\mathrm{CrGeTe}_{3}$ brought $2 \mathrm{D}$ magnetism into focus [23-25]. The FM ground state of bulk (multilayer) $\mathrm{CrI}_{3}$ displays a crucial dependence on the thickness. For instance, while its bilayer acquires an antiferromagnetic (AFM) ground state, the monolayer changes to become FM again [21]. Even more remarkable is that the $\mathrm{CrI}_{3}$ bilayer at fixed magnetic fields near the metamagnetic transition can switch from AFM state to FM state with applied gate voltage offering electrically tunable magnetism [26]. Presently, magnetism in $2 \mathrm{D}$ also showing tunability with decoration of magnetic ions [27], electrostatic doping [28,29], and many interesting magneto-optic and magnetoelectric effects [30-32] has been now a focus of interest.

\footnotetext{
*ge23xol@tum.de

†ciraci@fen.bilkent.edu.tr
}

Further to those recent discoveries in 2D magnetism, we report here another novel and interesting finding in 2D magnetism. Combining first-principles density functional theory (DFT) with Metropolis Monte Carlo (MC) calculations, we predict an unusual magnetic state, columnar -FM (CAFM) order in the $\mathrm{Fe}_{2} \mathrm{~B}_{2}$ monolayer with critical temperature $T_{c}=$ $115 \mathrm{~K}$. Below this temperature, the spins rotate by the same amount in every other column of $\mathrm{Fe}$ atoms, but they retain the same direction in the same column. Even more remarkable is that the critical temperature of this CAFM order can be changed and can even be raised under the tensile strain applied in reasonable ranges. While the rise of $T_{c}$ is not monotonic and depends on the type of strain, it can be as high as room temperature under a specific tensile strain. The onset of CAFM order below $T_{c}$ with the spins aligned in the same direction along the same column of $\mathrm{Fe}$ atoms, but periodically changing their directions in other columns may induce a transition in the electronic states from 2D to 1D, which may be reflected in the energy bands in the momentum space with specific quantized conductance behavior.

Under the applied magnetic fields, the spins rotate and eventually align along the direction of the field. At the end, the CAFM order changes to the FM order with characteristic double hysteresis. This feature makes the tunability of magnetic and electronic properties of the monolayer possible by externally applied magnetic field. Additionally, when $\mathrm{Fe}_{2} \mathrm{~B}_{2}$ is terminated by $\mathrm{F}$, its CAFM order can be changed to AFM order with $T_{c}$ above room temperature. We found that the competing exchange couplings between the nearest two $\mathrm{Fe}$ atoms in different planes underlie this CAFM order, which is novel, and have critical implications in 2D magnetism. 


\section{COMPUTATIONAL DETAILS}

We performed our theoretical analysis within firstprinciples plane-wave calculations based on spin-polarized DFT by using projected augmented wave (PAW) $[33,34]$ potentials to describe the ion-electron interaction, as implemented in the Vienna $A b$ initio Simulation Package $[35,36]$. For the exchange-correlation potential, generalized gradient approximation was adopted by using the PerdewBurke-Ernzerhof (PBE) [37] functional. The vdW correction within the method of Grimme (DFT-D2) [38] was taken into account. The energy cutoff of plane-wave basis was used as $500 \mathrm{eV}$. For the Brillouin Zone (BZ) integration in k-space, a set of $(21 \times 21 \times 1) \mathbf{k}$-point sampling was used within Monkhorst-Pack scheme [39] for unit cell calculations. The geometry optimization was achieved by using a conjugategradient algorithm [40,41] until the total energy and forces were minimized and the maximum pressure in the unit cell was reduced to less than $0.5 \mathrm{kbar}$. The energy convergence value was adjusted to $10^{-6} \mathrm{eV}$ between two successive electronic steps. The partial occupancies were set for each orbital by Methfessel-Paxton scheme [42] with a smearing width of $0.2 \mathrm{eV}$. Hybrid functional calculations were also performed over the PBE results [43]. For the charge-transfer analysis, Bader analysis [44] on a charge density grid was used. All of the structures were visualized in the VESTA code [45]. Spin-orbit coupling (SOC) is included in all calculations. In order to compute the phonon modes, we calculated force constants of the $(2 \times 2 \times 1)$ supercell in real space using density functional perturbation theory (DFPT) [46] as implemented in the PHONOPY [47] program. We also checked thermal stability of $\mathrm{Fe}_{2} \mathrm{~B}_{2}$ within $(2 \times 2 \times 1)$ supercell by performing ab initio molecular dynamics (AIMD) simulations at $500 \mathrm{~K}$ for 2 ps by using Nosé thermostat in 2-fs time steps. The exchange-coupling parameters could be calculated for different magnetic configurations using ground state energies obtained from first-principles calculations through the following equations: $J_{1}=\left(E_{\mathrm{AFM} 3}-E_{\mathrm{FM}}\right) / 12 M^{2} ; J_{2}=\left(E_{\mathrm{AFM} 1}-\right.$ $\left.E_{\mathrm{AFM} 2}\right) / 8 M^{2} ; J_{3}=\left(3 E_{\mathrm{AFM} 2}-2 E_{\mathrm{AFM} 3}-E_{\mathrm{FM}}\right) / 24 M^{2}$.

We have performed 100 independent realizations at each temperature. We implement Metropolis MC method [48-50] as the updating protocol for the present system. Initially, the simulation starts from a random configuration of spins at a temperature well above the critical temperature of the system, and in order to make a fine estimation of the transition temperature, the system has been progressively cooled down until the temperature reaches to $T=0.01 \mathrm{~K}$ with small temperature steps. During the simulations, we have monitored the quantities of interest over $10^{5} \mathrm{MC}$ steps per lattice site for equilibrium system, after discarding the first $5 \times 10^{4}$ steps.

\section{MAGNETIC PROPERTIES OF $\mathrm{Fe}_{2} \mathrm{~B}_{2}$ MONOLAYER}

Earlier, free-standing metallic $\mathrm{Fe}_{2} \mathrm{~B}_{2}$ monolayers were first predicted theoretically and its stability was assured by Guo et al. [19]. More recently, Liu et al. [51] reported the synthesis of the parent MAB phase, $\mathrm{Fe}_{2} \mathrm{AlB}_{2}$, using solid phase sintering. The isolation of the ultrathin film or monolayer of $\mathrm{Fe}_{2} \mathrm{~B}_{2}$ from the parent 3D MAB phase by etching or by molecular beam epitaxy has been proposed [19,51-53]. In (a)

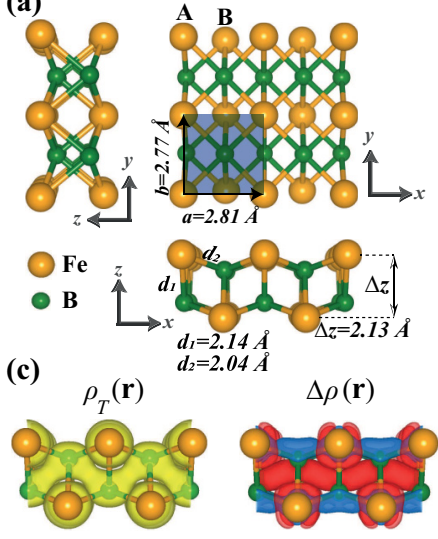

(b)

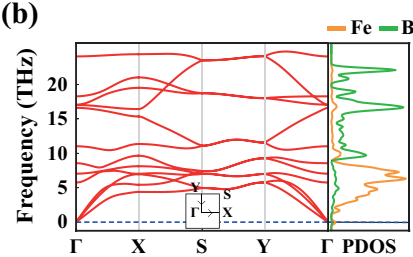

FIG. 1. (a) Structure of the $\mathrm{Fe}_{2} \mathrm{~B}_{2}$ monolayer: Top (in the $x y$ plane) and side views (in the $z y$ and $z x$ planes) of optimized atomic configurations of orthorhombic $\mathrm{Fe}_{2} \mathrm{~B}_{2}$ with 2D rectangular lattice and $a / b \approx 1$. Two $\mathrm{Fe}$ atoms in the unit cell reside in two different atomic planes separated by $\Delta z=2.13 \AA$. The $2 \mathrm{D}$ unit cell is shaded; the lattice constants and relevant structural parameters are shown. (b) Phonon dispersion curves along major symmetry directions of the 2D BZ and atom-projected densities of states (PDOS). (c) Isosurfaces of total charge density, $\rho_{T}(\mathbf{r})$, and difference charge density, $\Delta \rho(\mathbf{r})$ Excess electronic charge is indicated in blue. (d) The electronic energy band structure, $E_{n}(\mathbf{k})$, calculated within PBE and HSE along major symmetry directions of the 2D BZ and related PDOS, indicates metallic state. Zero of energy is set at the Fermi level shown by the black, dashed line.

particular, the synthesis of a 2D MBene through topochemical deintercalation of $\mathrm{Al}$ from a similar MAB phase has been achieved successfully [54].

To test the capacity of the first-principles method and the parameters therein in predicting magnetic properties, we first calculated the optimized atomic structure of $3 \mathrm{D}$ parent orthorhombic $\mathrm{Fe}_{2} \mathrm{AlB}_{2}$ phase to be $\mathrm{a}=2.89 \AA$, $\mathrm{b}=10.86 \AA$, and $\mathrm{c}=2.80 \AA$, which are in agreement with experiments [55]. Among various possible magnetic orders, we predicted a robust FM ground state with spins oriented along the $a$ axis as found experimentally [56]. Furthermore, the calculated magnetic moment of $1.27 \mu_{B} / \mathrm{Fe}$ and magnetic anisotropy energies also agree with experiments [57].

\section{A. Atomic and Electronic Structure}

Having been assured from the accuracy of the method to be used in the forthcoming analysis concerning the magnetic state of the $\mathrm{Fe}_{2} \mathrm{~B}_{2}$ monolayer, we now examine the stability of its atomic structure optimized with the present parameters and the corresponding electronic energy bands for the sake of consistency. As described in Fig. 1(a), the optimized atomic structure has 2D rectangular lattice and its unit cell comprises two $\mathrm{Fe}$ atoms in different atomic planes separated by $\Delta z=$ $2.13 \AA$ A. Even if its high cohesive energy of $22.4 \mathrm{eV}$ per unit cell indicates an enhanced binding, its stability is verified through dynamical and thermal stability analysis performed by including SOC. As shown in Fig. 1(b), positive phonon frequencies over the $\mathrm{BZ}$ even for $\mathbf{k} \rightarrow 0$ demonstrate that the $\mathrm{Fe}_{2} \mathrm{~B}_{2}$ monolayer is not prone to instability. Our AIMD 
calculations also assure that the suspended monolayer can sustain thermal excitations above room temperature at $T=$ $500 K$. In addition, calculated in-plane stiffness values $\left(C_{x}=182\right.$ and $\left.C_{y}=158 \mathrm{~J} / \mathrm{m}^{2}\right)$ indicate that the monolayer possesses rather high mechanical strength with each $\mathrm{Fe}(\mathrm{B})$ atom having six nearest $\mathrm{B}(\mathrm{Fe})$ atoms.

Isosurfaces of the total electronic charge density $\rho_{T}(\mathbf{r})$ in Fig. 1(c) show how $3 d$-orbitals are localized at $\mathrm{Fe}$ atoms. In the $y z$ plane of $\mathrm{B}$ atoms, one sees that $\rho_{T}(\mathbf{r})$ is high, indicating the formation of bonds between $\mathrm{B}$ atoms in different planes. The difference charge density $\Delta \rho(\mathbf{r})$, which is obtained by subtracting free atom charge densities situated at the atomic sites from $\rho_{T}(\mathbf{r})$, shows the character of charge transfer. According to Bader analysis [44], 0.34 electrons are transferred from $\mathrm{Fe}$ atoms to B atoms. Electronic energy band structure presented in Fig. 1(d) is calculated with PAW potential within PBE [37] by including SOC. Several bands originated from $\mathrm{Fe}-3 d$ orbitals cross the Fermi level, leading to a rather high density of states at the Fermi level, $D\left(E_{F}\right)$. Accordingly, the $\mathrm{Fe}_{2} \mathrm{~B}_{2}$ monolayer is a magnetic metal. Calculations based on hybrid functional, HSE [43], which is known to predict the band gap correctly, also confirmed the metallic state. Notably, the recent angle-resolved photoemission spectroscopy data reveal high electron density near $E_{F}$ induced by linear bands originating from $\mathrm{Fe}-d_{x y}$ orbitals and imply a complex electronic-magnetic correlation effect with non-Fermi liquid behavior in $3 \mathrm{D} \mathrm{Fe}_{2} \mathrm{AlB}_{2}$ [51]. Incidentally, $\mathrm{Fe}_{2} \mathrm{~B}_{2}$ monolayer dominates the electronic structure of the parent, layered $\mathrm{Fe}_{2} \mathrm{AlB}_{2}$ phase. By performing self-consistent electronic structure calculations for each tensile strain considered in this work, we assured that the metallic state is also preserved under strain. More details can be found in the supplemental material [58].

Next we study the magnetic properties of the $\mathrm{Fe}_{2} \mathrm{~B}_{2}$ monolayer, which is the prime objective of our study. For the sake of consistency, we will use the same method and calculational parameters in the above analysis. The spin Hamiltonian within the classical Heisenberg model given below is a crucial step in our analysis of magnetic properties:

$$
\begin{aligned}
\mathcal{H}=- & J_{1} \sum_{\langle i, j\rangle} \vec{\mu}_{i} \cdot \vec{\mu}_{j}-J_{2} \sum_{\langle i, k\rangle} \vec{\mu}_{i} \cdot \vec{\mu}_{k}-J_{3} \sum_{\langle i, l\rangle} \vec{\mu}_{i} \cdot \vec{\mu}_{l} \\
& -\sum_{i}\left(k_{x} \mu_{i x}^{2}+k_{y} \mu_{i y}^{2}+k_{z} \mu_{i z}^{2}\right)-\sum_{i} \vec{B} \cdot \vec{\mu}_{i},
\end{aligned}
$$

where, respectively, $J_{1}, J_{2}$, and $J_{3}$ are exchange couplings indicated schematically in Fig. 2(a) and $\vec{B}$ is the magnetic field vector. The values of these exchange coupling parameters are retrieved from the total energies corresponding to four different magnetic configurations realized in the $(2 \times 2 \times 1)$ supercell of the $\mathrm{Fe}_{2} \mathrm{~B}_{2}$ monolayer as described in Fig. 2(b), which were calculated from first-principles within DFT. Their values are found to be $J_{1}=29.92, J_{2}=4.12$, and $J_{3}=$ $-16.16 \mathrm{meV}$ for unstrained lattice (i.e., $\epsilon=0$ ). The values calculated for the lattice under diverse types of strains are given in the supplemental material [58]. The dynamical stabilities of these four different configurations, all in metallic state, were verified through phonon calculations. The fourth term in Eq. (1) represents the single ion anisotropy energy of the system; $\vec{\mu}_{i}$ is the classical magnetic dipole moment located

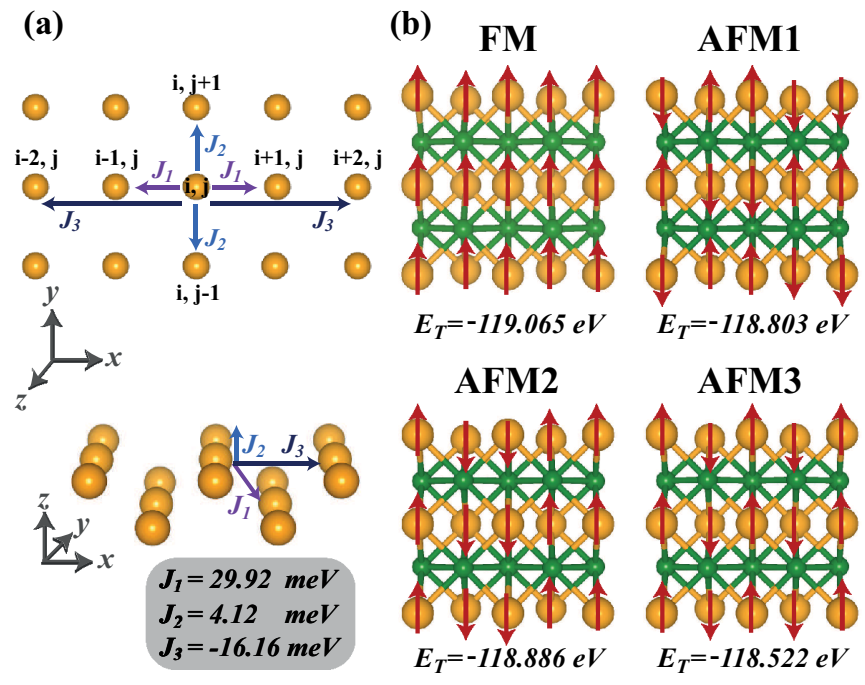

FIG. 2. (a) Top: Schematic description of exchange coupling parameters $J_{1}, J_{2}$, and $J_{3}$ in the superlattice used in MC calculations. Columns of $\mathrm{Fe}$ atoms along the $y$ direction are numbered by $i ; j$ is the row index along the $x$ direction. Bottom: Columns of $\mathrm{Fe}$ atoms along the $y$ direction in the upper and lower atomic planes of $\mathrm{Fe}_{2} \mathrm{~B}_{2}$ monolayer are shown in a perspective view. Calculated values of exchange coupling parameters are also given. (b) Four spatial spin configurations (one FM and three AFM, AFM1, AFM2, AFM3) in the $(2 \times 2 \times 1) \quad \mathrm{Fe}_{2} \mathrm{~B}_{2}$ supercell with their calculated total energies $E_{T}$.

at the sites of a 2D square $(L=100 \times L=100)$ superlattice used in MC calculations.

The FM configuration, which is realized in the $(2 \times$ $2 \times 1)$ supercell, appears to be a lowest energy configuration among four different configurations in Fig. 2 and attains $10.67 \mu_{B}$ per supercell magnetic moment derived from $\mathrm{Fe}-3 d$ orbitals. The magnetic anisotropy energies $\left(E_{M A}\right)$ are calculated by rotating all spins to different directions according to the expression $E_{M A}=E_{u v w}-E_{\min }$, where $E_{\min }$ is the energy of the most stable spin orientation. The calculated in-plane $E_{M A}$ (i.e., E[100]-E[010]) is $\sim-1.4 \mathrm{meV}$, whereas out-ofplane $E_{M A}$ (i.e., E[100]-E[001]) is $\sim 641 \mu \mathrm{eV}$. Very small $E_{M A}$ energies obtained from the difference of the energies' corresponding two different spin directions are sensible, as also reported in recent studies [59] and imply that $\mathrm{Fe}_{2} \mathrm{~B}_{2}$ monolayer has only small in-plane magnetic anisotropy.

We performed MC calculations using the classical Heisenberg model, where each spin is represented by vectors of magnitude $3 / 2$ (for zero strain) residing on the sites $(i\|x ; j\| y)$ of the $(L=100 \times L=100)$ superlattice; $i$ $(j)$ is specified as column (row) index. Initially, these vectors are randomly oriented. Spin-spin interaction occurs as described in Fig. 2(a) in terms of exchange coupling parameters discussed in the foregoing paragraph. These calculations provided us for the variations of order parameter $(\mathbf{O}=$ $\left.\frac{1}{L L !}\left\langle\sum_{i=1}^{L} \sum_{j=1}^{L} \sum_{k=j+1}^{L} \vec{\mu}_{i j} \cdot \vec{\mu}_{i j+k}\right\rangle\right)$ and heat capacity $(\mathbf{C}=$ $\left.\left\langle\frac{\partial \mathcal{H}}{\partial T}\right\rangle\right)$ of $\mathrm{Fe}_{2} \mathrm{~B}_{2}$ monolayer with temperature $T$. In Fig. 3, we present the variation of the order parameter $\mathbf{O}$, heat capacity $\mathbf{C}$, and $T_{c}$ with temperature and applied (biaxial $\epsilon_{x y}$, uniaxial $\epsilon_{x}$ and $\epsilon_{y}$ ) tensile strains up to $10 \%$. 
(a)
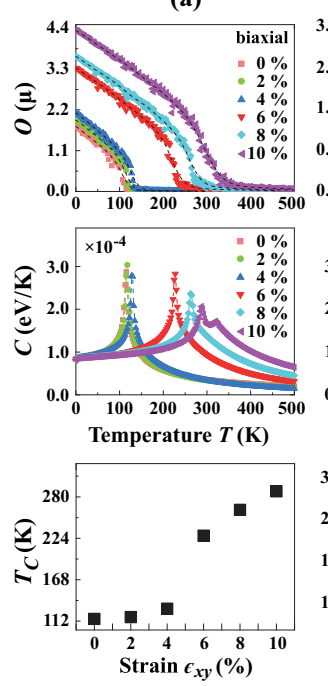

(b)
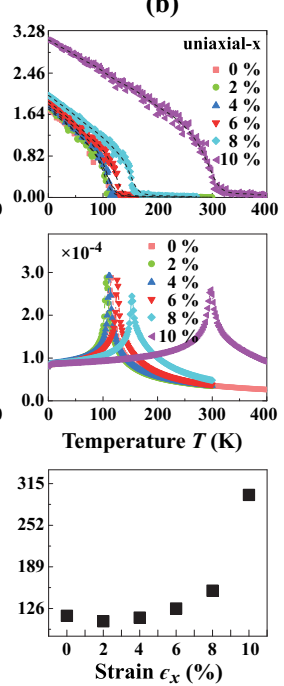

(c)
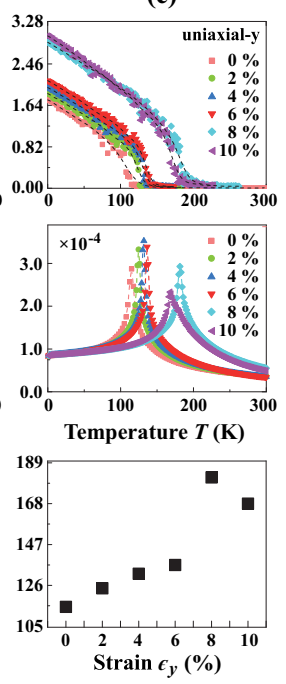

FIG. 3. (a) Variation of the order parameter $\mathbf{O}$ and heat capacity $\mathbf{C}$ in zero applied magnetic field with temperature $T$ for various biaxial tensile strain values $\epsilon_{x y}$, and the variation of critical temperature $T_{c}$ with biaxial strain, $\epsilon_{x y}$. (b) Same for uniaxial strain $\epsilon_{x}$ along the $x$ direction. (c) Same for the uniaxial strain $\epsilon_{y}$ along the $y$ direction.

The peak value of the heat capacity of unstrained monolayer marks the critical temperature for the order-disorder transition to be $T_{c}=115 \mathrm{~K}$. This indicates a magnetic order in $\mathrm{Fe}_{2} \mathrm{~B}_{2}$ monolayer, which is sustainable at finite temperature. As $T$ decreases from $T_{c}$, the magnetic order increases and eventually the order parameter saturates to $\mu^{2}$ value. As depicted in Fig. 3, critical temperature and hence onset of order-disorder transition change with applied strain. In general, the saturation value of the order parameter increases with increasing tensile strain, since the magnitude of the magnetic dipole moment $\vec{\mu}$ increases. As for the critical temperature of the monolayer under strain, it does not display a common behavior; rather, it depends on the type and range of the applied tensile strain. For example, $T_{c}$ is not affected up to $\epsilon_{x y} \leqslant \% 4$, but it increases suddenly when $\epsilon_{x y}>\% 5$, like an "S" curve. As expected, the variation of $T_{c}$ in the range $0 \leqslant$ $\epsilon_{x} \leqslant \% 10$ and $0 \leqslant \epsilon_{y} \leqslant \% 10$ displays different behaviors in Figs. 3(b) and 3(c), since the atomic structure of the monolayer differs along $x$ and $y$ directions as depicted in Fig. 1(a). It appears that $T_{c}$ can rise to the room temperature as $\epsilon_{x} \rightarrow \% 10$, which is, nevertheless, a rather high elastic deformation. That the critical temperature can rise with applied tensile strain is the first important result of our study.

\section{B. Columnar AFM Order}

Further to these magnetic properties, our MC simulations predict that the ground state of $\mathrm{Fe}_{2} \mathrm{~B}_{2}$ monolayer has a CAFM order not only in equilibrium (i.e., $\epsilon=0$ ) but also under the applied tensile strain as described above. This is the second important result. Even if earlier studies have found columnar magnetic ordering in some 3D bulk structures, the present prediction of CAFM in a strictly $2 \mathrm{D}$ and realistic monolayer like $\mathrm{Fe}_{2} \mathrm{~B}_{2}$ with rectangular lattice is a novel finding. As shown in Figs. 4(a)-4(c), this ordered phase is constructed at (a)
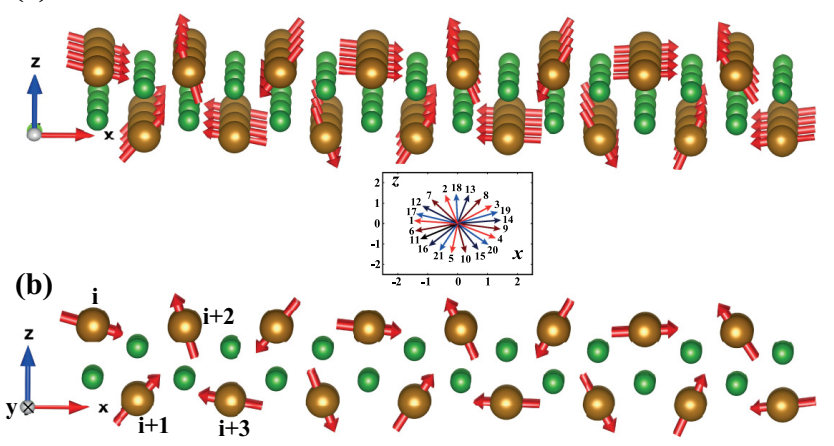

(c)

(d)

(e)

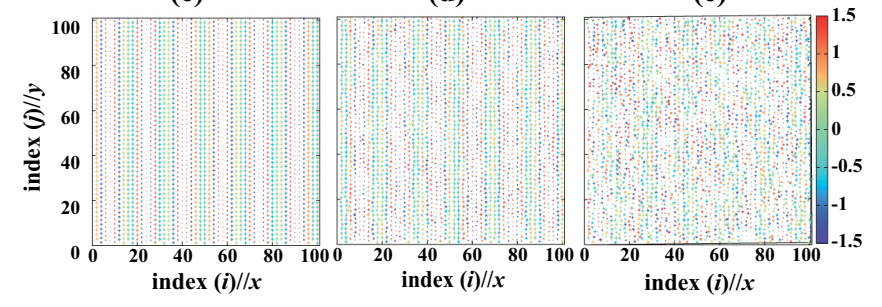

FIG. 4. Spatial spin configurations simulated at different temperatures for $\epsilon=0$. (a) A perspective top view of the monolayer with Fe (large gold balls) and B (small green balls) atoms. The direction of spins and hence the $x$ and $z$ components of spins, which are kept invariant along an individual column $i$ (made of Fe atoms along the $y$ direction) through sites $1 \leqslant j \leqslant 100$, but they vary in subsequent columns $1 \leqslant i \leqslant 100$. The $y$ components of the spins are negligible. (b) Side view in the $x z$ plane. Rotation of spins in the $x$ and $z$ plane with increasing column index $i$ is depicted by inset, whereby each column is translated to the same center. (c) Perfect CAFM order at $T=0.01 K$ is depicted in the superlattice $(i \times j)$. In color codes, spin components range from $-3 / 2$ to $3 / 2$. (d) Same for $T=50.0 \mathrm{~K}$. (e) Same for the disordered phase at $T=200.0 \mathrm{~K}$.

low temperature in individual columns of the regular lattice, whereby a spin in a particular site $(i, j)$ is perfectly aligned with its neighbors $(i, j-1)$ and $(i, j+1)$ in the same column along the $y$ direction because of $J_{2}>0$. Here each column is constructed by either $\mathrm{Fe}$ atoms along the $y$ direction in the upper atomic plane or the adjacent, parallel column of $\mathrm{Fe}$ atoms in the lower atomic plane as shown in Fig. 3. Concurrently, as if a spin wavelike mechanism is perpendicular to the columns of $\mathrm{Fe}$ atoms, the direction of spins rotates gradually in the $x$ and $z$ planes with increasing column index, $i$, so that their directions in nearby columns rotate even if the spins in each column are perfectly aligned to have the same direction. Stated differently, spins rotate in the $x z$ plane by the same amount by going from one column to the next, but they keep the same direction in the same column. The alignment of spins is in the same column, but their gradual rotation with (i) is shown in Figs. 4(a) and 4(b) in perspective top and side atomistic views, respectively. The rotation of spins with increasing $i$ to follow a circle in the $x z$ plane is described in the inset, whereby all columns are translated to the same center. Notably, the $y$ components of spins are negligible. Accordingly, while the spin direction is positive initially, it decreases gradually and eventually is reversed with increasing $i$ as described by color codes in Fig. 4(c). Moreover, this 
situation repeats itself showing a spatial periodicity as $i$ progresses. Hence, the spin direction of one column is recovered approximately after five-six columns. We believe that such an interesting magnetic order is realized as a consequence of the atomic structure of $\mathrm{Fe}_{2} \mathrm{~B}_{2}$ monolayer consisting of two planes of $\mathrm{Fe}$ atoms, which constitute adjacent columns of Fe in different planes with a nearest intercolumn distance of $d<b<a$. As $J_{1}$, being positive, favors parallel alignments of spins between nearest lateral sites, $J_{3}$, being negative, forces them in the antiparallel alignment between the next nearest lateral sites (see Fig. 2). Hence the competitions of exchange couplings between the nearest and the next nearest Fe columns give rise to periodic, CAFM order. Moreover, this CAFM order is a robust property for all strains studied here, since the order parameters at low temperature increase with increasing strain as depicted in Fig. 3. We also note that this ground state acquired from MC simulations comprising large degrees of freedom in a large supercell differs from DFT calculation performed only for a very limited number (four) of magnetic configurations in a comparatively very small $(2 \times 2$ $\times 1$ ) supercell of $\mathrm{Fe}_{2} \mathrm{~B}_{2}$ monolayer. How the perfect CAFM order with periodically varying spin direction at $T=0.01 \mathrm{~K}$ is destroyed with increasing temperature is shown in Figs. 4(d) and 4(e).

It is known that the classical Heisenberg model results in different ground states when second- or third-nearestneighbor exchange interactions are included [60]. For instance, a system represented by a classical Heisenberg model on 2D (hexagonal and triangular) lattices considering only the nearest- and second-nearest-neighbor interactions may be not only in FM and AFM phases but also in triangular formation and helical ordering, depending on the values of exchange interactions [61]. Néel, spiral, dimerized, and other possible critical phases for the $J_{1}-J_{2}$ Heisenberg model have also been discussed [62]. A honeycomb lattice, treated with the classical Heisenberg $J_{1}-J_{2}-J_{3}$ model, can then exhibit columnar and spiral phases $[60,63]$. Furthermore, the classical Heisenberg model on the Shastry-Sutherland lattice could display spin-flip, spin-flop, and umbrella phases $[64,65]$. Even though most of these studies have been devoted to 2D honeycomb lattices, Heisenberg $J_{1}-J_{2}$ [62] and Heisenberg $J_{1}-J_{2}-J_{3}$ models [66] on an abstract, square lattice [66] have just started. Also, it has been demonstrated that bilayer systems may also exhibit interesting configurations including noncoplanar states and spiral phases [67]. Notably, quantum models also reveal a wide variety of the phases including spiral ordering, valence-bond crystalline phases with nematic ordering, and spin liquid phases [68-72].

To clarify whether the CAFM order predicted by the PBE method with PAW potential can be an artifact of this method, we also repeated the above DFT $+\mathrm{MC}$ calculations by also using PW-91 functional [73] and PBE functional but two different potentials with GW and PV corrections [36]. All three calculations, which resulted in the CAFM ground state with $T_{c}$ differing only $\pm 7 \mathrm{~K}$ from that of our original prediction, assured that our conclusions are rather robust. Furthermore, similar DFT $+\mathrm{MC}$ calculations on $\mathrm{Gd}_{2} \mathrm{~B}_{2}$ have been very successful in reproducing experimental observations concerning its magnetic ground state and transition temperature [74].
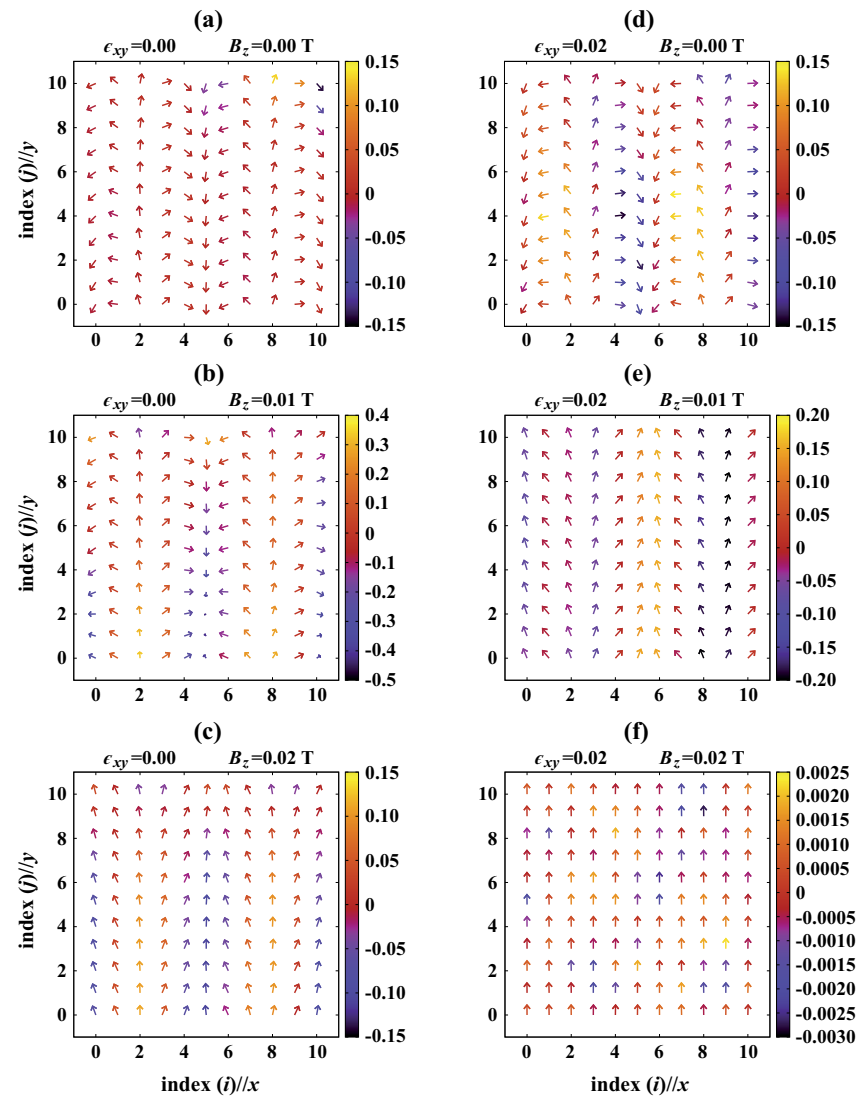

FIG. 5. Spatial spin configurations of the $\mathrm{Fe}_{2} \mathrm{~B}_{2}$ monolayer at the temperature $T=0.01 \mathrm{~K}$ are shown in a small grid of $i=1-10$ and $j=1-10$ for $\epsilon=0$ and $\epsilon_{x y}=0.02$ under the applied magnetic field, $\vec{B}$ parallel to the $z$ axis in diverse magnitudes (in Tesla, T). Arrows indicate the spin directions in the $x z$ plane, where $x \| i$; color codes correspond to the the $y$ component of the spins.

\section{Transition from CAFM to FM Order under Magnetic Field}

The behavior of the CAFM order under a magnetic field applied externally is a crucial feature, which may provide a tool to tune electrical and magnetic properties. The expectation is that the spins, which are in CAFM order along the columns of $\mathrm{Fe}$ atoms as depicted in Fig. 4, can be forced to change their directions and to align along the applied magnetic field where the degree of alignment is proportional to the strength of the field. Whether an hysteresis is involved with the alignment process is another important aspect to reveal. With these premises, we investigated the response of the ordered system to the applied magnetic field vector $\vec{B}$ in different directions with components $\left(B_{x}, 0,0\right),\left(0, B_{y}, 0\right)$, and $\left(0,0, B_{z}\right)$ at $T=0 \mathrm{~K}$.

Our MC calculations show that relatively small values of the field are actually sufficient to align the spins parallel to the field at low temperatures. Our results are presented in Fig. 5 only for $\vec{B}\left(0,0, \mathrm{~B}_{z}\right)$ with $B_{z}=0.00,0.01$ and 0.02 Tesla ( $\mathrm{T}$ ) applied perpendicular to the $\mathrm{Fe}_{2} \mathrm{~B}_{2}$ monolayer under the strain $\epsilon=0$ and $\epsilon_{x y}=0.02$. Although the change of the original spin configuration by the applied magnetic field is strain dependent [compare, for instance, Figs. 5(c) with 5(f)], all spin configurations corresponding to the different strain 

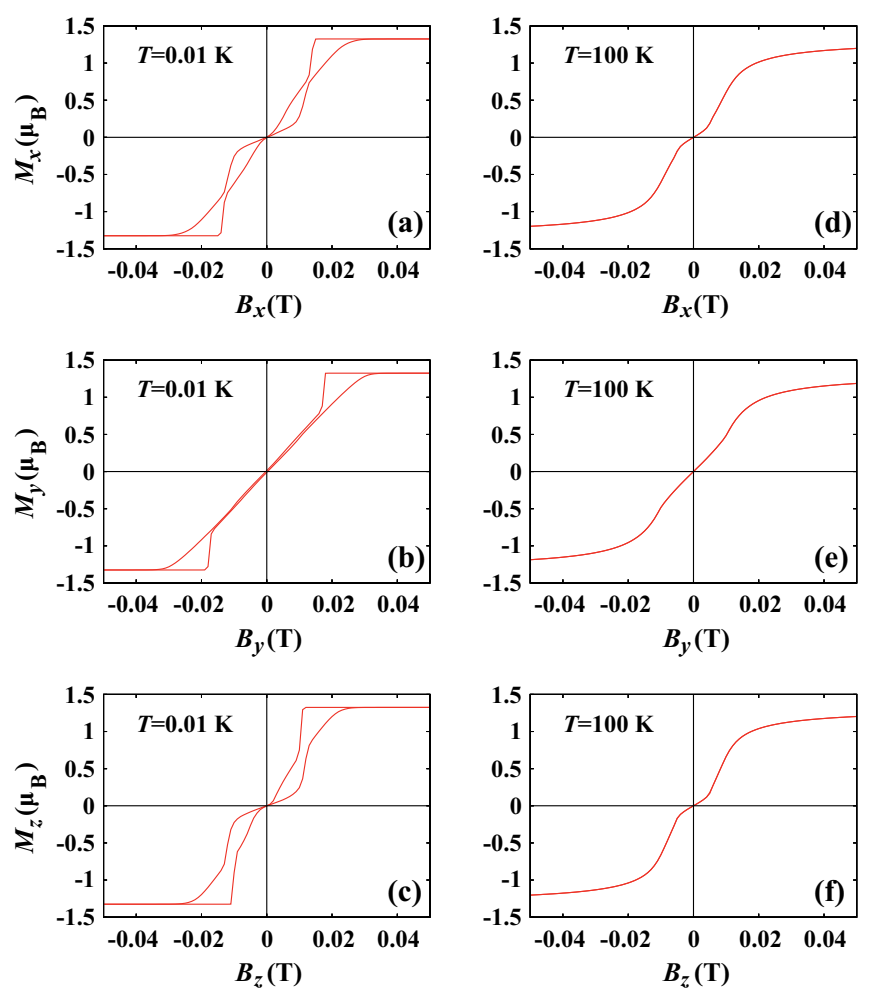

FIG. 6. Hysteresis curves, $M_{\alpha}$ (in unit of Bohr magneton $\mu_{B}$ per site) versus $B_{\alpha}$ (in unit of Tesla $\mathrm{T}$ ), of the unstrained $\mathrm{Fe}_{2} \mathrm{~B}_{2}$ monolayer calculated for the external magnetic fields applied along $\alpha=x, y, z$ directions at $T=0.01 \mathrm{~K}$ and $T=100.0 \mathrm{~K}$.

values quickly reach the field polarized spin configuration already at $B \approx 0.03 \mathrm{~T}$, so the system reaches to the saturation value of the magnetization. The present prediction is important and heralds the possibility that the magnetic properties of the $\mathrm{Fe}_{2} \mathrm{~B}_{2}$ monolayer can be tuned externally; namely that its CAFM order with zero total magnetic moments occurring at low temperature can be changed to a uniform FM order of spins with strong total magnetic moment (and magnetization) along the applied magnetic field. This is the third important result of our study.

The hysteresis of the unstrained $\mathrm{Fe}_{2} \mathrm{~B}_{2}$ monolayer, namely, $M_{\alpha}$ versus $B_{\alpha}$ curves are calculated for applied magnetic fields in directions $\alpha=x, y, z$, with components $\left(B_{x}, 0,0\right)$, $\left(0, B_{y}, 0\right)$, and $\left(0,0, B_{z}\right)$ at temperatures $T=0.01 K$ and $T=100.0 \mathrm{~K}$. It should be noted that due to the CAFM order, all components of the magnetization are zero for $\vec{B}=$ 0 at low temperatures. However, only the component $M_{\alpha}$ is induced in response to the applied magnetic field, $B_{\alpha}$, while other components of the magnetization vanish. Calculations are performed in a small superlattice or a grid of $(80 \times 80)$ for each strain value. First, the system is cooled down to the temperature $T=0.01 \mathrm{~K}$ by the MC procedure explained above, then simulations are performed by sweeping the field $B_{\alpha}$ and obtaining the components of the magnetization $M_{\alpha}$. The related hysteresis curves are shown in Fig. 6. Notably, hysteresis curves of different strain values treated in this paper display qualitatively similar behaviors in Fig. 6 . The double hysteresis behavior occurs in Figs. 6(a)-6(c) due to the absence of magnetization for $\vec{B}=0$. Nonetheless, this double (a)
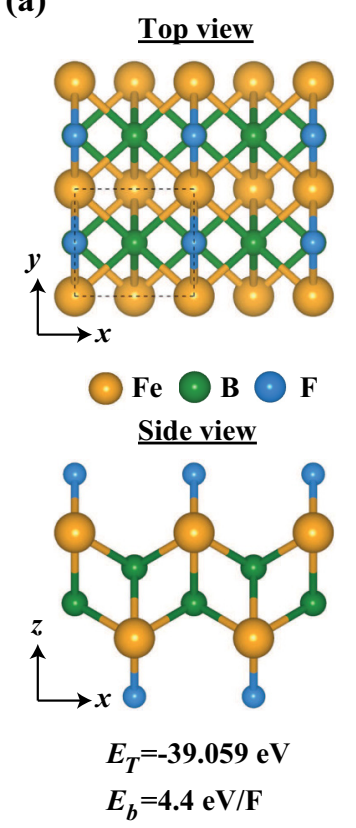

(b)

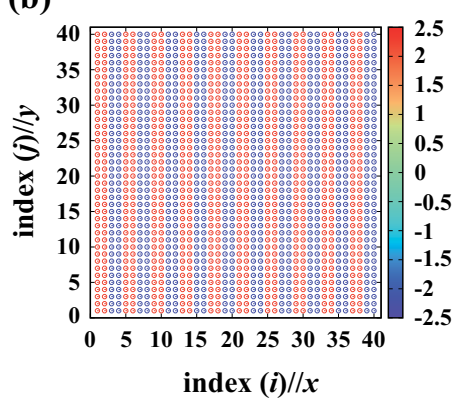

(c)

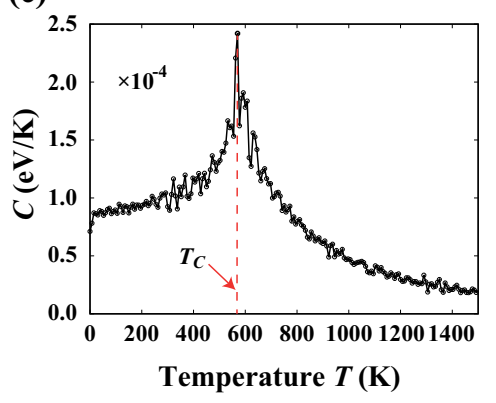

FIG. 7. Fluorine-covered $\mathrm{Fe}_{2} \mathrm{~B}_{2}$ monolayer: $\mathrm{Fe}_{2} \mathrm{~B}_{2} \mathrm{~F}_{2}$. (a) Top and side views of the equilibrium binding structure with $\mathrm{F}$ atoms situated at the hollow sites above $\mathrm{B}$ atoms between $\mathrm{Fe}$ atoms in the upper and lower sides. The total and binding energies corresponding to the optimized geometry are indicated. (b) MC results showing the $y$ component of spins of in the $(i \times j)$ grid at $T=0.01 K$ indicate that AFM-2 configuration is the ground state. Other components are negligible. (c) Variation of the heat capacity $\mathrm{C}$ with temperature. The transition temperature $T_{c}$ above $500 \mathrm{~K}$ is indicated.

hysteresis behavior disappears due to thermal agitations at elevated temperatures as seen in Figs. 6(d)-6(f).

\section{Transition from CAFM to AFM by Adatom Coverage}

Finally, the magnetic ground state of the $\mathrm{Fe}_{2} \mathrm{~B}_{2}$ monolayer terminated by adatoms like Fluorine $\mathrm{F}$, which are also ubiquitous among MBenes, is of interest. Notably, it was argued that the coverage of $\mathrm{F}$ is critical in the isolation of the monolayer from the $3 \mathrm{D}$ MAB phase, $\mathrm{Fe}_{2} \mathrm{AlB}_{2}$. The equilibrium binding site of individual $\mathrm{F}$ atoms on the monolayer has been determined by calculating optimized total energies corresponding to five possible different binding sites. Among these sites, we found that the hollow site above B atoms at each side is the minimum energy site with the binding energy of $E_{b}=4.40 \mathrm{eV}$. As shown in Fig. 7(a), F adatoms form bridge bonds with adjacent $\mathrm{Fe}$ atoms in the column along $y$ axis with a bond length of $1.94 \AA$. The calculated charge transfer of 1.08 electrons from the $\mathrm{Fe}_{2} \mathrm{~B}_{2}$ monolayer indicates an ionic bonding. Our analysis based on AIMD calculations demonstrated that this F-covered monolayer, i.e., $\mathrm{Fe}_{2} \mathrm{~B}_{2} \mathrm{~F}_{2}$, is thermally stable above room temperature as high as $T=600 \mathrm{~K}$. MC calculations, using the $J_{1}, J_{2}$, and $J_{3}$ exchange couplings obtained from four different magnetic configurations described in Fig. 2, predict the AFM-2 order shown in Fig. 7(b) as the ground state, which is $\sim 0.4 \mathrm{eV}$ more favorable energetically relative to other magnetic configurations. Variation of the heat capacity calculated by the MC method in Fig. 7(c) marks the critical 
temperature, $T_{c} \geqslant 500 \mathrm{~K}$. It is remarkable that the coverage of the bare $\mathrm{Fe}_{2} \mathrm{~B}_{2}$ monolayer by Fluorine atoms changes the exchange coupling parameters, which, in turn, changes the CAFM order to the AFM order and raises $T_{c}$ above $500 \mathrm{~K}$. This is the fourth important result of our study.

\section{CONCLUSION}

In conclusion, the CAFM order with transition temperature $T_{c}=115 \mathrm{~K} \pm 7 \mathrm{~K}$, confirmed by four different calculations, unveils novel and important features in the magnetism of $2 \mathrm{D}$ materials. We believe that the observation of CAFM order can be achieved by Magnetic Force Microscopy, which enables the atomic scale probe of magnetism through its sharp magnetic tip.

Spins aligned along each column of $\mathrm{Fe}$ atoms as indicated in Figs. 4(a) and 4(b), but kept different directions at different columns, bring about the following fundamental question concerning the dimensionality of electrons: Can the dimensionality of electrons in the paramagnetic monolayer at high temperature change from 2D to 1D on the onset of CAFM order below $T_{c}$ ? We believe that this is a rather sensible question to raise, since the electrons with different spin alignments in adjacent 1D Fe columns may be distinguished to lead to different 1D electronic states. This situation can favor the transition of the electronic states from 2D to 1D with the onset of CAFM order. Such an electronic transition can become observable through the change in electronic densities $D(E)$ of states, as well as quantized 1D conductance along Fe columns $\sigma$. Theoretically, it may be interesting to know how 1D electrons with specific spin direction in each Fe column is reflected in the energy bands in the momentum space. We believe that the observation of this electronic transition induced by the onset of CAFM order and its tuning by applied magnetic field $\vec{B}$, strain $\epsilon$, and adatom coverage can be achieved experimentally. From a technological point of view, tuning of the magnetic order and the dimensionality of quantized conductance can lead to significant effects in spintronics. In the same context, it is important to know whether spins aligned along the same column, but periodically changing their direction in different columns, can be distinguished to enable multichannel spintronic devices. On the other side, CAFM phase of the bare $\mathrm{Fe}_{2} \mathrm{~B}_{2}$ monolayer, with $T_{c}$ rising up to room temperature under specific tensile strains and AFM phases sustaining at $T_{c}$ as high as $600 \mathrm{~K}$ on Fluorine termination, can emphatically lead to future spin-based devices and detectors.

\section{ACKNOWLEDGMENTS}

The computational resources are provided by TUBITAK ULAKBIM, High Performance and Grid Computing Center (TR-Grid e-Infrastructure). This work was supported by the Scientific and Technological Research Council of Turkey (TUBITAK) under the Research Project No. 118F354. S.C. acknowledges financial support of Academy of Science of Turkey (TÜBA). E.A. acknowledges financial support of the Alexander von Humboldt Foundation (Germany).
[1] K. S. Novoselov, A. K. Geim, S. V. Morozov, D. Jiang, Y. Zhang, S. V. Dubonos, I. V. Grigorieva, and A. A. Firsov, Electric field effect in atomically thin carbon films, Science 306, 666 (2004).

[2] E. Durgun, S. Tongay, and S. Ciraci, Silicon and III-V compound nanotubes: Structural and electronic properties, Phys. Rev. B 72, 075420 (2005).

[3] S. Cahangirov, M. Topsakal, E. Aktürk, H. Şahin, and S. Ciraci, Two- and One-Dimensional Honeycomb Structures of Silicon and Germanium, Phys. Rev. Lett. 102, 236804 (2009).

[4] P. Vogt, P. De Padova, C. Quaresima, J. Avila, E. Frantzeskakis, M. C. Asensio, A. Resta, B. Ealet, and G. Le Lay, Silicene: Compelling Experimental Evidence for Graphenelike Two-Dimensional Silicon, Phys. Rev. Lett. 108, 155501 (2012).

[5] Y. Xu, B. Yan, H. J. Zhang, J. Wang, G. Xu, P. Tang, W. Duan, and S. C. Zhang, Large Gap Quantum Spin Hall Insulators in Tin Films, Phys. Rev. Lett. 111, 136804 (2013).

[6] H. Şahin, S. Cahangirov, M. Topsakal, E. Bekaroglu, E. Aktürk, R. T. Senger, and S. Ciraci, Monolayer honeycomb structures of group-IV elements and III-V binary compounds: Firstprinciples calculations, Phys. Rev. B 80, 155453 (2009).

[7] D. Kecik, A. Onen, M. Konuk, E. Gürbüz, F. Ersan, S. Cahangirov, E. Aktürk, E. Durgun, and S. Ciraci, Fundamentals, progress, and future directions of nitride-based semiconductors and their composites in two-dimensional limit: A first-principles perspective to recent synthesis, Appl. Phys. Rev. 5, 011105 (2018).
[8] P. Joensen, R. F. Frindt, and S. R. Morrison, Single-layer $\mathrm{MoS}_{2}$, Mater. Res. Bull. 21, 457 (1986).

[9] K. S. Novoselov, D. Jiang, F. Schedin, T. J. Booth, V. V. Khotkevich, S. V. Morozov, and A. K. Geim, Two-dimensional atomic crystals, Proc. Natl. Acad. Sci. USA 102, 10451 (2005).

[10] J. N. Coleman, M. Lotya, A. O'Neill, S. D. Bergin, P. J. King, U. Khan, K. Young, A. Gaucher, S. De, R. J. Smith et al., Two-dimensional nanosheets produced by liquid exfoliation of layered materials, Science 331, 568 (2011).

[11] K. F. Mak, C. Lee, J. Hone, J. Shan, and T. F. Heinz, Atomically Thin $\mathrm{MoS}_{2}$ : A New Direct-Gap Semiconductor, Phys. Rev. Lett. 105, 136805 (2010).

[12] C. Ataca, H. Şahin, and S. Ciraci, Stable, single-layer $\mathrm{MX}_{2}$ transition-metal oxides and dichalcogenides in a honeycomblike structure, J. Phys. Chem. C 116, 8983 (2012).

[13] Y. Li, K. A. N. Duerlo, K. Wauson, and E. J. Reed, Structural semiconductor-to-semimetal phase transition in twodimensional materials induced by electrostatic gating, Nat. Commun. 7, 10671 (2016).

[14] S. Demirci, H. H. Gürel, S. Cahangirov, and S. Ciraci, Temperature, strain and charge mediated multiple and dynamical phase changes of selenium and tellurium, Nanoscale 12, 3249 (2020).

[15] V. O. Ozcelik, S. Cahangirov, and S. Ciraci, Stable SingleLayer Honeycomblike Structure of Silica, Phys. Rev. Lett. 112, 246803 (2014).

[16] Z. Zhu and D. Tomanek, Semiconducting Layered Blue Phosphorus: A Computational Study, Phys. Rev. Lett. 112, 176802 (2014). 
[17] C. Kamal and M. Ezawa, Arsenene: Two-dimensional buckled and puckered honeycomb arsenic systems, Phys. Rev. B 91, 085423 (2015).

[18] F. Ersan, D. Keçik, V. O. Özçelik, Y. Kadioglu, O. Üzengi Aktürk, E. Durgun, E. Aktürk, and S. Ciraci, Two-dimensional pnictogens: A review of recent progresses and future research directions, Appl. Phys. Rev. 6, 021308 (2019).

[19] Z. Guo, J. Zhou, and Z. Sun, New two-dimensional transition metal borides for Li ion batteries and electrocatalysis, J. Mater. Chem. A 5, 23530 (2017).

[20] Y. Ma, Y. Dai, M. Guo, C. Niu, Y. Zhu, and B. Huang, Evidence of the existence of magnetism in pristine $\mathrm{VX}_{2}$ monolayers $(\mathrm{X}=\mathrm{S}, \mathrm{Se})$ and their strain-induced tunable magnetic properties, ACS Nano 6, 1695 (2012).

[21] B. Huang, G. Clark, E. Navarro-Moratalla et al., Layerdependent ferromagnetism in a van der Waals crystal down to the monolayer limit, Nature 546, 270 (2017).

[22] C. Gong, L. Li, Z. Li et al., Discovery of intrinsic ferromagnetism in two-dimensional van der Waals crystals, Nature 546, 265 (2017)

[23] C. Gong and X. Zhang, Two-dimensional magnetic crystals and emergent heterostructure devices, Science 363, eaav4450 (2019).

[24] K. S. Burch, D. Madrus, and J. G. Park, Magnetism in twodimensional van der Waals materials, Nature 563, 47 (2018).

[25] M. Gibertini, M. Koperski, A. F. Morpurgo, and K. S. Novoselov, Magnetic 2D materials and heterostructures, Nat. Nanotechnol. 14, 408 (2019).

[26] B. Huang, G. Clark, D. R. Klein et al., Electrical control of 2D magnetism in bilayer $\mathrm{CrI}_{3}$, Nat. Nanotechnol. 13, 544 (2018).

[27] A. M. Tokmachev, D. V. Averyanov, O. E. Parfenov et al., Emerging two-dimensional ferromagnetism in silicene materials, Nat. Commun. 9, 1672 (2018).

[28] S. Jiang, J. Shan, and K. F. Mak, Electric-field switching of twodimensional van der Waals magnets, Nat. Mater. 17, 406 (2018).

[29] S. Jiang, L. Li, Z. Wang, K. F. Mak, and J. Shan, Controlling magnetism in $2 \mathrm{D} \mathrm{CrI}_{3}$ by electrostatic doping, Nat. Nanotechnol. 13, 549 (2018).

[30] T. Song, X. Cai, M. W. Y. Tu et al., Giant tunneling magnetoresistance in spin-filter van der Waals heterostructures, Science 360, 1214 (2018).

[31] K. L. Seyler, D. Zhong, D. R. Klein et al., Ligand-field helical luminescence in a 2D ferromagnetic insulator, Nat. Phys. 14, 277 (2018).

[32] D. R. Klein, D. MacNeill, J. L. Lado et al., Probing magnetism in $2 \mathrm{D}$ van der Waals crystalline insulators via electron tunneling, Science 360, 1218 (2018).

[33] P. E. Blöchl, Projector augmented-wave method, Phys. Rev. B 50, 17953 (1994).

[34] G. Kresse and D. Joubert, From ultrasoft pseudopotentials to the projector augmented-wave method, Phys. Rev. B 59, 1758 (1999).

[35] G. Kresse and J. Furthmüller, Efficiency of ab-initio total energy calculations for metals and semiconductors using a plane-wave basis set, Comput. Mater. Sci. 6, 15 (1996).

[36] G. Kresse and J. Furthmüller, Efficient iterative schemes for ab initio total-energy calculations using a plane-wave basis set, Phys. Rev. B 54, 11169 (1996).

[37] J. P. Perdew, K. Burke, and M. Ernzerhof, Generalized Gradient Approximation Made Simple, Phys. Rev. Lett. 77, 3865 (1996).
[38] S. Grimme, Semiempirical GGA-type density functional constructed with a long-range dispersion correction, J. Comput. Chem. 27, 1787 (2006).

[39] H. J. Monkhorst and J. D. Pack, Special points for Brillouinzone integrations, Phys. Rev. B 13, 5188 (1976).

[40] C. G. Broyden, The convergence of a class of double-rank minimization algorithms: 1. General considerations, IMA J. Appl. Math. 6, 76 (1970).

[41] C. G. Broyden, The convergence of a class of double-rank minimization algorithms: 2. The new algorithm, IMA J. Appl. Math. 6, 222 (1970).

[42] M. Methfessel and A. T. Paxton, High-precision sampling for Brillouin-zone integration in metals, Phys. Rev. B 40, 3616 (1989).

[43] J. Heyd, G. E. Scuseria, and M. Ernzerhof, Hybrid functionals based on a screened Coulomb potential, J. Chem. Phys. 118, 8207 (2003)

[44] G. Henkelman, A. Arnaldsson, and H. Jonsson, A fast and robust algorithm for Bader decomposition of charge density, Comput. Mater. Sci. 36, 354 (2006).

[45] K. Momma, and F. Izumi, VESTA 3 for three-dimensional visualization of crystal, volumetric and morphology data, J. Appl. Crystallogr. 44, 1272 (2011).

[46] S. Baroni, S. de Gironcoli, A. Dal Corso, and P. Giannozzi, Phonons and related crystal properties from density-functional perturbation theory, Rev. Mod. Phys. 73, 515 (2001).

[47] A. Togo and I. Tanaka, First principles phonon calculations in materials science, Scr. Mater. 108, 1 (2015).

[48] D. P. Landau and K. Binder, A Guide to Monte Carlo Simulations in Statistical Physics (Cambridge University Press, 2014).

[49] M. Newman and G. Barkema, Monte Carlo Methods in Statistical Physics (Oxford University Press, New York, 1999), Vol. 24, Chap. 1-4.

[50] G. Marsaglia, Choosing a point from the surface of a sphere, Ann. Math. Stat. 43, 645 (1972).

[51] Z. Liu, S. Yang, H. Su et al., Non-fermi-liquid behavior and saddlelike flat band in the layered ferromagnet $\mathrm{AlFe}_{2} \mathrm{~B}_{2}$, Phys. Rev. B 101, 245129 (2020).

[52] H. Zhang, H. Xiang, F. Z. Dai, Z. Zhang, and Y. Zhou, First demonstration of possible two-dimensional MBene $\mathrm{CrB}$ derived from MAB phase $\mathrm{Cr}_{2} \mathrm{AlB}_{2}$, J. Mater. Sci. \& Technol. 34, 2022 (2018)

[53] Z. Jiang, P. Wang, X. Jiang, and J. Zhao, MBene (MnB): A new type of 2D metallic ferromagnet with high Curie temperature, Nanoscale Horiz. 3, 335 (2018).

[54] L. T. Alameda, P. Moradifar, Z. P. Metzger, N. Alem, and R. E. Schaak, Topochemical deintercalation of Al from MoAlB: Stepwise etching pathway, layered intergrowth structures, and two-dimensional MBene, J. Am. Chem. Soc. 140, 8833 (2018).

[55] M. ElMassalami, D. D. S. Oliveira, and H. Takeya, On the ferromagnetism of $\mathrm{AlFe}_{2} \mathrm{~B}_{2}$, J. Magn. Magn. Mater. 323, 2133 (2011).

[56] J. Cederwall, M. S. Anderson, T. Sarkar et al., Magnetic structure of the magnetocaloric compound $\mathrm{AlFe}_{2} \mathrm{~B}_{2}$, J. Alloys Compd. 664, 784 (2016).

[57] T. N. Lamichhane, L. Xiang, Q. Lin et al., Magnetic properties of single crystalline itinerant ferromagnet $\mathrm{AlFe}_{2} \mathrm{~B}_{2}$, Phys. Rev. Materials 2, 084408 (2018).

[58] See Supplemental Material at http://link.aps.org/supplemental/ 10.1103/PhysRevB.103.144424 for additional informations, 
such as electronic energy band structures calculated for each tensile strain considered in this work, optical properties of $\mathrm{Fe}_{2} \mathrm{~B}_{2}$ monolayer, and snap shots of atomic structures obtained from the finite temperature ab initio molecular dynamics calculations for the $\mathrm{Fe}_{2} \mathrm{~B}_{2}$ monolayer at 300 and $500 \mathrm{~K}$ temperatures, etc., which includes Refs. [19,75-77].

[59] L. Ciorciaro, M. Kroner, K. Watanabe, T. Taniguchi, and A. Imamoglu, Observation of Magnetic Proximity Effect Using Resonant Optical Spectroscopy of an Electrically Tunable MoSe2/CrBr3 Heterostructure, Phys. Rev. Lett. 124, 197401 (2020).

[60] E. Rastelli, A. Tassi, and L. Reatto, Non-simple magnetic order for simple Hamiltonians, Physica B+C 97, 1 (1979).

[61] S. Katsura, T. Ide, and T. Morita, The ground states of the classical heisenberg and planar models on the triangular and plane hexagonal lattices, J. Stat. Phys. 42, 381 (1986).

[62] Z. Weihong, R. H. McKenzie, and R. R. P. Singh, Phase diagram for a class of spin-1/2 Heisenberg models interpolating between the square-lattice, the triangular-lattice, and the linear-chain limits, Phys. Rev. B 59, 14367 (1999).

[63] J. B. Fouet, P. Sindzingre, and C. Lhuillier, An investigation of the quantum J1-J2-J3 model on the honeycomb lattice, Eur. Phys. J. B 20, 241 (2001).

[64] A. Grechnev, Exact ground state of the Shastry-Sutherland lattice with classical Heisenberg spins, Phys. Rev. B 87, 144419 (2013).

[65] M. Moliner, D. C. Cabra, A. Honecker, P. Pujol, and F. Stauffer, Magnetization process in the classical Heisenberg model on the Shastry-Sutherland lattice, Phys. Rev. B 79, 144401 (2009).

[66] S. Rufo, J. R. de Sousa, and J. A. Plascak, Exchange frustration effect due to anisotropies on the spin-1/2 J1-J1'-J2 model, Physica A 518, 349 (2019).

[67] R. F. Bishop and P. H. Y. Li, Frustrated honeycomb-bilayer Heisenberg antiferromagnet: The spin-1/2 J1-J2-J1' model, Phys. Rev. B 95, 134414 (2017).
[68] R. F. Bishop and P. H. Y. Li, Complete phase diagram of the spin-1/2 J1-J2-J3 model (with J3=J2) on the honeycomb lattice, Phys. Rev. B 85, 155135 (2012).

[69] P. H. Y. Li, R. F. Bishop, D. J. J. Farnell, J. Richter, and C. E. Campbell, Ground-state phases of the frustrated spin-1/2 J1-J2J3 Heisenberg ferromagnet $(\mathrm{J} 1<0)$ on the honeycomb lattice with J3=J2 > 0, Phys. Rev. B 85, 085115 (2012).

[70] P. H. Y. Li, R. F. Bishop, D. J. J. Farnell, and C. E. Campbell, Phase diagram of a frustrated Heisenberg antiferromagnet on the honeycomb lattice: The J1-J2-J3 model, Phys. Rev. B 86, 144404 (2012).

[71] A. Kalz, M. Arlego, D. Cabra, A. Honecker, and G. Rossini, Anisotropic frustrated Heisenberg model on the honeycomb lattice, Phys. Rev. B 85, 104505 (2012).

[72] J. Oitmaa and R. R. P. Singh, Phase diagram of the J1-J2-J3 Heisenberg model on the honeycomb lattice: A series expansion study, Phys. Rev. B 84, 094424 (2011).

[73] J. P. Perdew and Y. Wang, Erratum: Accurate and simple analytic representation of the electron-gas correlation energy [Phys. Rev. B 45, 13244 (1992)], Phys. Rev. B 98, 079904(E) (2018).

[74] T. Gorkan, E. Vatansever, Ü. Akıncı, G. Gokoglu, E. Aktürk, and S. Ciraci, Above room temperature ferromagnetism in Gd2B2 monolayer with high magnetic anisotropy, J. Phys. Chem. C 124, 12816 (2020).

[75] R. C. Andrew, R. E. Mapasha, A. M. Ukpong, and N. Chetty, Mechanical properties of graphene and boronitrene, Phys. Rev. B 85, 125428 (2012).

[76] M. Elahi, K. Khaliji, S. M. Tabatabaei, M. Pourfath, and R. Asgari, Modulation of electronic and mechanical properties of phosphorene through strain, Phys. Rev. B 91, 115412 (2015).

[77] Z. Guo, J. Zhou, C. Si, and Z. Sun, Flexible two dimensional $\mathrm{Ti}_{n+1} \mathrm{C}_{n}$ ( $\mathrm{n}=1,2$ and 3) and their functionalized MXenes predicted by density functional theories, Phys. Chem. Chem. Phys. 17, 15348 (2015). 